\title{
What You Should Know About NAMD and Charm++ But Were Hoping to Ignore
}

\author{
James C. Phillips \\ National Center for Supercomputing Applications \\ University of Illinois at Urbana-Champaign \\ Urbana, Illinois \\ jcphill@illinois.edu
}

\begin{abstract}
The biomolecular simulation program NAMD is used heavily at many HPC centers. Supporting NAMD users requires knowledge of the Charm++ parallel runtime system on which NAMD is built. Introduced in 1993, Charm++ supports message-driven, task-based, and other programming models and has demonstrated its portability across generations of architectures, interconnects, and operating systems. While Charm++ can use MPI as a portable communication layer, specialized high-performance layers are preferred for Cray, IBM, and InfiniBand networks and a new OFI layer supports Omni-Path. NAMD binaries using some specialized layers can be launched directly with mpiexec or its equivalent, or mpiexec can be called by the charmrun program to leverage system job-launch mechanisms. Charm++ supports multi-threaded parallelism within each process, with a single thread dedicated to communication and the rest for computation. The optimal balance between thread and process parallelism depends on the size of the simulation, features used, memory limitations, nodes count, and the core count and NUMA structure of each node. It is also important to enable the Charm++ built-in CPU affinity settings to bind worker and communication threads appropriately to processor cores. Appropriate execution configuration and $\mathrm{CPU}$ affinity settings are particularly non-intuitive on Intel KNL processors due to their high core counts and flat NUMA hierarchy. Rules and heuristics for default settings can provide good default performance in most cases and dramatically reduce the search space when optimizing for a specific simulation on particular machine. Upcoming Charm ++ and NAMD releases will simplify and automate launch configuration and affinity settings.
\end{abstract}

\section{CCS CONCEPTS}

- Social and professional topics $\rightarrow$ Computing equipment management; Software selection and adaptation; Computer and information systems training; • Applied computing $\rightarrow$ Molecular structural biology; Chemistry; Physics; • Computing methodologies $\rightarrow$ Parallel programming languages;

ACM acknowledges that this contribution was authored or co-authored by an employee, contractor, or affiliate of the United States government. As such, the United States government retains a nonexclusive, royalty-free right to publish or reproduce this article, or to allow others to do so, for government purposes only.

PEARC '18, fuly 22-26, 2018, Pittsburgh, PA, USA

(C) 2018 Association for Computing Machinery.

ACM ISBN 978-1-4503-6446-1/18/07 . \$ \$15.00

https://doi.org/10.1145/3219104.3219134

\section{KEYWORDS}

NAMD, Charm++, structural biology, molecular dynamics, highperformance computing, scientific software tuning, user support

\section{ACM Reference Format:}

James C. Phillips. 2018. What You Should Know About NAMD and Charm++ But Were Hoping to Ignore. In PEARC '18: Practice and Experience in $A d-$ vanced Research Computing, July 22-26, 2018, Pittsburgh, PA, USA. ACM, New York, NY, USA, 6 pages. https://doi.org/10.1145/3219104.3219134

\section{INTRODUCTION}

An underlying goal during the two decades that NAMD and Charm++ have been co-developed has been to isolate the user (and in the case of Charm ++ , the programmer), from the details of achieving efficient parallel scaling. In particular, it is intended that a NAMD simulation can be run on any platform and number of processors without any modification to the input files. This consistency has resulted in a popular and scientifically productive code that has retained much of its internal architecture across many generations of high-performance computing (HPC) platforms.

The variety of hardware and software environments on which NAMD is used, however, requires choices to be made both when building Charm++ and NAMD, and when selecting the command arguments used to launch NAMD in parallel on the machine. The selection of the most performant build and launch options unfortunately requires at least rough knowledge of the intended simulation sizes and enhanced sampling methods to be used. This paper provides guidance on making appropriate choices.

\section{INTENDED AUDIENCE}

This paper is directed at HPC professionals responsible for supporting NAMD users. It should be of value to anyone interested in ensuring the efficiency of NAMD simulations, particularly on distributed memory clusters and supercomputers. Both beginning and experienced NAMD users are likely to learn something new, as this material has not been previously collected in a single reference.

\section{NAMD AND ITS USERS}

All diseases are the result of the disruption of the functioning of cells at the molecular level, and all drugs act by either restoring balance to the molecules of the body or disrupting those of invading bacteria and viruses [5]. Molecular dynamics (MD) simulations enable the study of cellular processes at atomic and nanosecond resolution not accessible via experiment, but are limited in size and duration by computational resources. 
NAMD [6] is one of many MD codes specialized for biomolecular systems, each with features and capabilities driven by the research interests of its developers. Recent NAMD development has focused on exploiting GPU acceleration $[7,10]$ and petascale supercomputing [9], and on integrating data from new forms of microscopic imaging.

The typical NAMD user is a biophysical or biochemical domain scientist with limited interest and experience in programming or parallel computing. NAMD provides cross-platform extensibility through scripting in $\mathrm{Tcl}$ [8], a language users are familiar with from its use in the molecular visualization program VMD, allowing custom simulation protocols to be implemented without recompiling the program. Tcl scripting has enabled several methodological contributions by NAMD users.

The NAMD user community is large and diverse. The NAMD 2.12 release was downloaded by over 13,000 unique registrants, and the NAMD reference paper is cited over 1,200 times per year. In a survey of NAMD users, $43 \%$ report a high level of molecular modeling expertise and at least $69 \%$ of users are familiar with other MD programs, yet $57 \%$ primarily use NAMD, and $79 \%$ say their scientific productivity would suffer without it.

NAMD is highly used at the NSF supercomputing centers, and is the most-used code on NCSA's Blue Waters petascale system. The NAMD developers are currently engaged in early science preparations for the 2018 Oak Ridge Summit POWER9/Volta cluster, and for the 2021 Argonne Aurora exascale system. The successful application of these expensive machines to important biomedical problems is impossible without assistance from HPC professionals.

\section{NAMD/CHARM++ LICENSE TERMS}

The following is the author's opinion based on his personal experience and understanding and should not be relied on as either legal advice or as representing the official views of the University of Illinois.

NAMD and Charm++ are distributed free of charge as both source code and pre-compiled binaries under nearly identical custom licenses designed to enable the respective development teams to track usage of the software in order to justify funding for continued development and support. To this end, redistribution is prohibited and NAMD users are required to register when downloading the software and to cite a specific paper [6] in resulting publications.

Common cases that are not considered prohibited redistribution are installing NAMD on your (organization's) cluster, and installing NAMD on someone else's cluster for use by yourself and members of your organization. The license also grants explicit permission to develop complementary software that inter-operates with NAMD, to distribute patches to the NAMD source code, and to freely re-use up to $10 \%$ of the NAMD source code in other programs containing at least an equal amount of new code.

Commercial entities are allowed to use NAMD for internal research purposes under the standard license. The prominent prohibition on "commercial use" applies actually to commercialization such as incorporating NAMD into a product or service.

\section{THE CHARM++ RUNTIME SYSTEM}

NAMD is based on the Charm++ parallel programming system. Unlike traditional statically mapped and decomposed message-passing programming, Charm++ uses message-driven execution and an adaptive runtime system (RTS) to manage the mapping of work to processors $[1,3]$. The RTS adds flexibility to the distribution of work by combining automatic load balancing with over-decomposition. Over-decomposition also enables the overlap of computation and communication by increasing the likelihood that there are other objects ready to execute while waiting for messages to be received over the network.

NAMD cannot be built without Charm++, but this has not limited NAMD's portability as Charm++ is co-developed with NAMD and is often ported to emerging platforms either by the vendor or during a friendly user period. Charm ++ can be built on MPI for portability to new high performance interconnects, although specialized machine layers generally provide higher performance. The launch mechanism for non-MPI layers varies by platform, but system-provided utilities for launching MPI jobs are leveraged to interface with the batch scheduler. Users of laptops and desktops enjoy pre-compiled binaries for single-process execution and IP (UDP, TCP) or InfiniBand (verbs) parallel communication. Future Charm++ portability is being enhanced by migrating machine layer implementations to a common low-level runtime system (LRTS), reducing the amount of redundant code.

Charm++ uses an unconventional internal nomenclature that may appear in NAMD startup and error messages. A Charm++ "PE" (processing element) is a worker thread (typically a POSIX thread running on a dedicated hardware thread). A Charm++ "node" is a process (a set of PEs sharing a memory space). A Charm++ "physical node" is a host (running a set of Charm++ nodes that share network interfaces and GPUs, and which are assumed to communicate faster among themselves than with other hosts).

\section{BUILD PROCESS OVERVIEW}

The NAMD build process requires judgments that would be unreliable to automate, but is otherwise straightforward. In particular, one must select the compiler, an appropriate inter-node communication target, and between single-threaded and multi-threaded ("smp") processes. Multiple versions (e.g., both smp and non-smp) may be desirable to better support different classes of simulations. Charm++ must first be built for a particular platform and compiler, and then NAMD built based on that platform and with the same compiler. A compatible and tested version of Charm++ is included with all NAMD source code distributions. In addition to Charm++, NAMD requires Tcl and FFTW (serial, single-precision, version 2 or 3) libraries, which are commonly installed on HPC systems or are available for download as pre-compiled libraries on the NAMD website. The NVIDIA CUDA toolkit is required for GPU acceleration. NAMD does not support a formal installation process, as the namd2 and other binaries are mostly self-contained and users tend to run them in-place by specifying a complete path to the build or distribution directory.

\section{CHARM++ BUILD OPTIONS}

The Charm++ build script, run without options, will default to an interactive mode in which it attempts to detect the target platform and asks the user to confirm defaults or choose from alternatives before initiating the build process. Each build with different options 
will create a correspondingly named sub-directory, e.g., verbs-linuxx86_64-icc. The same build can be done non-interactively by specifying the target $($ charm ++$)$ and platform on the build command line, e.g.,./build charm++ verbs-linux-x86_64-icc --no-build-shared --with-production -j8”. Note that platform options such as the compiler can be specified separated by spaces rather than hyphens, e.g., "netlrts-linux-x86_64 tcp smp icc", and will be reordered by the build script to construct the hyphenated build directory. A full list of options can be obtained via "./build --help". The Charm++ CUDA infrastructure is not used by NAMD and should not be enabled. Modifications to the build commands that cannot be set via build-line options may require editing the corresponding .sh files in the src/arch subdirectories.

Supported Charm++ communication targets include multicore (multi-threaded single process), netlrts (UDP), netlrts tcp (TCP), verbs (InfiniBand), ofi (Omni-Path via Open Fabrics Interfaces), crayxe-gni (Gemini), crayxc-gni (Aries), and mpi (system MPI library). The "smp" multi-threading option is supported on all platforms except multicore (which is multi-threaded by default). Multicore NAMD binaries are the most efficient on a single node, but non-smp binaries are generally more efficient than smp binaries for typical simulation sizes on small node counts. Running an smp build with a single thread per process is not an alternative to a nonsmp build as smp builds have a dedicated communication thread. Further, NAMD will exit on startup if an smp build is run singlethreaded as failing to specify the thread count on the command line is a common user error. NAMD builds with CUDA GPU acceleration require an smp build of Charm++ and the NAMD config script will reject MPI-smp for CUDA builds due to poor MPI-smp performance. Note that deprecated pre-LRTS communication targets, such as net and net-ibverbs, do not support multi-copy algorithms in NAMD.

\section{NAMD BUILD OPTIONS}

NAMD is built by using the config script to specify options and create a build directory, and then running "make release" in that directory to build and package the binaries, text files, and lib directory. Running the config script without arguments will print a list of options and suggested platform-compiler targets, such as Linux-x86_64-g++. Available targets and their default compiler options are defined by .arch make include files in the arch subdirectory, e.g., arch/Linux-x86_64-g++.arch, with optional compileragnostic features in .tcl, .fftw, .fftw3, .cuda, etc. make include files, e.g., arch/Linux-x86_64.tcl. In order to support build scripting, library paths and other compile-line options can be specified as config script arguments, but it is occasionally necessary or convenient to edit the files in the arch directory. The Make.charm file can be edited to provide a default path to the Charm++ compilation directory, but it is ignored if the config script detects a Charm++ directory in the NAMD distribution directory.

A NAMD config script command line could be as simple as “./config Linux-x86_64-g++", which would create the build directory Linux-x86_64-g++ containing a Make.config file and various symbolic links, relying on default values in the arch files. Alternatively, the build directory name can be given an extension and various options customized, e.g., "./config Linux-x86_64-icc.cuda_verbs -charm-base /path/to/charm --charm-arch verbs-linux-x86_64-smpiccstatic --with-fftw3 --tcl-prefix /path/to/tcl-threaded --with-cuda --cuda-prefix /usr/local/cuda-9.1"

\section{LAUNCH MECHANISMS}

Every Charm++ build includes, depending on the platform, a binary or script named "charmrun" that serves to launch the application across nodes, the equivalent of mpiexec or mpirun for MPI applications. For multicore builds, the provided script is unnecessary as the namd2 binary can be run directly, specifying the number of threads with $+\mathrm{p} n$. Builds based on MPI, Cray GNI, OFI, and IBM PAMI are launched the same as are MPI programs on the machine (mpirun, mpiexec, ibrun, aprun, jsrun, etc.).

For smp builds on the above platforms, the number of worker threads (PEs) per process must be specified as +ppn threads, e.g., "mpiexec -n 4 namd 2 +ppn 7 ..." would launch 4 processes, each with 7 worker threads plus a communication thread, thus using a total of 32 cores (or hardware threads). Care must be taken to specify to the platform launch system the total number of threads (worker plus communication) for each process so that sufficient cores are reserved and affinity set, otherwise all of the NAMD threads may end up sharing a single core. Note that the PAMI and multicore platforms lack a separate communication thread and may thus use all cores for computation. When running multi-copy algorithms with NAMD, if each replica is a single process then the communication thread will sleep when idle and thus also does not require a dedicated core.

A binary charmrun is provided for the netlrts and verbs platforms, as well as the older net platform from which they are descended, and charmrun is necessary to launch multi-process runs for these platforms. Command-line options processed by charmrun begin with ++ , those processed by the Charm ++ runtime begin with + , and all others are reserved for the application. Options not processed by charmrun must appear on the command line after the application binary. The option $+\mathrm{p} n$ is processed by charmrun as well, and specifies the total number of worker threads (PEs) across all processes in the job, which for smp builds must be a multiple of the number of threads per process as specified with +ppn.

For multi-process runs on a single host, "charmrun ++local" will launch processes directly on the calling host via the fork() system call. For multi-host runs, while charmrun can accept a list of hosts in its own format and ssh to them directly to launch processes, this is not recommended as it is difficult to interface with queueing systems. The charmrun ++mpiexec option will use mpiexec to launch processes on other nodes, with the processes then establishing socket connections back to charmrun and udp/tcp/verbs parallel communication amongst themselves. If the mpiexec command does not accept the $-\mathrm{n}$ option (and instead obtains the process count directly from environment variables set by the queueing system) then ++ mpiexec-no-n should be substituted. An MPI launch command other than mpiexec, either as used on the system or a wrapper script, may be specified by ++ remote-shell command. For example, on the original TACC Stampede the launch command was "charmrun +pn ++mpiexec-no-n ++remote-shell ibrun namd2 ..." Finally, if it is necessary to set environment variables not otherwise 
propagated, a wrapper script to be called on the remote hosts, taking as its arguments the application binary and arguments, may be specified as ++runscript script.

\section{CPU AFFINITY}

NAMD will generally run faster if each thread (or process for nonsmp builds) is restricted to run on a single core or hardware thread. Process affinity is now commonly set by mpiexec or other launch utilities, but this is often not functional for Charm++ programs so it is more reliable to use the Charm++-provided mechanisms, which are consistent across platforms, and further to directly specify the mapping to Charm++. The recommended Charm++ options to be passed to NAMD are +pemap and, for smp builds, +commap as well, specifying respectively the cores to be used for PEs and communication threads. Each accepts a list of CPU sets with the format "first [-last[:stride[.run $]][+$ span[...][,...]". For example, for hosts with two 16-core Intel processors running 4 processes per host, one could use “+ppn 7 + pemap 0-31:8.7 + commap 7-31:8" to place communication threads on cores 7,15,23,31 and PEs on cores 0-6,8-14,16-22,24-30. To also interleave PEs on hyperthreads, which on Intel processors are not grouped consecutively, one would use “+ppn 14 +pemap 0-31:8.7+32 +commap 7-31:8”. One should be careful to verify that cores are not oversubscribed, ideally with the utility top, and to validate that performance is improved.

In order to simplify the process of selecting and specifying CPU affinities, as well as the number of processes per host and threads per process, Charm++ is being adapted to use the hwloc library to discover processor configuration and make appropriate choices. The + pemap and +commap commands will, however, remain as at least a necessary fallback, in particular for rare processors such as Intel Xeon Phi Knights Landing (KNL) or IBM POWER.

\section{XEON PHI KNIGHTS LANDING}

Systems based on the KNL processor deserve special mention due to their large core and thread counts $(64,68$, or 72 cores with 4 hardware threads each). KNL binaries should always be built with the Intel processor with the flag -xMIC-AVX512 to optimize for the wide vector units. NAMD includes Linux-KNL-icc and CRAYXC-KNL-intel arch files that also define NAMD_KNL to enable optimized AVX512 kernels. Charm++ can be compiled for Cray Aries systems as "build charm++ gni-crayxc persistent smp -xMICAVX512"; and for Intel Omni-Path clusters as either "build charm++ ofi-linux-x86_64 smp icc-xMIC-AVX512" or "build charm++ mpilinux-x86_64 smp icc -xMIC-AVX512" as the observed performance difference between the OFI and MPI machine layers has been limited thus far. Both Cray and Omni-Path systems require multiple processes per node in order to drive the network, but Cray requires fewer as more of the communication processing is offloaded to the network adapter than on Omni-Path.

The logic in selecting a launch configuration and CPU affinity for $\mathrm{KNL}$ is guided by the following principles. Leave at least one core free to isolate operating system noise. Since pairs of cores on a "tile" share a 1MB L2 cache, do not split a tile between PEs of different nodes, but possibly split a tile between comm threads as they have different data access patterns than PEs. Use 1 or 2 hyperthreads for PE cores, but dedicate a core to each comm thread to reduce communication bottlenecks Multiple communication threads are required per host, although fewer for Cray Aries and than for Intel Omni-Path, with the added benefit that multiple copies of static data reduce memory bandwith contention.

Different KNL models accommodate different launch configurations. On Argonne Theta with 64 cores, less one for the OS (aprun -r 1), the following configurations fit: $63=9 \times 7=9 \times(6+1)=54$ $\mathrm{PE}+9$ comm, affinity “+ppn 12 + pemap 0-53+64 + commap 54-62”; $63=7 \times 9=7 \times(8+1)=56 \mathrm{PE}+7$ comm, affinity “+ppn $16+$ pemap $0-55+64+$ commap 56-62"; and $60=4 \times 15=4 \times(14+1)=56 \mathrm{PE}+4$ comm, affinity “+ppn 28 +pemap 0-63:16.14+64 + commap 14-62:16”. On TACC Stampede2, with 68 cores, the following are possible: $65=13 \times 5=13 \times(4+1)=52 \mathrm{PE}+13$ comm, affinity “+ppn 8 +pemap 0-51+68 + commap 53-65”; $66=6 \times 11=6 \times(10+1)=60$ $\mathrm{PE}+6$ comm, affinity “+ppn 20 + pemap 0-59+68 + commap 60-65”; and $68=4 \times 17=4 \times(16+1)=64 \mathrm{PE}+4$ comm, affinity " + ppn 32 +pemap 0-63+68 +commap 64-67". The recommended defaults are 7 processes per node on Theta (aprun $-\mathrm{n} \$\left(\left(7^{*}\right.\right.$ \$nodes $\left.)\right)-\mathrm{N} 7-\mathrm{d} 17-\mathrm{j} 2$ -r 1) and 13 processes per node on Stampede2, although production simulations should be benchmarked on the target node count to select the best configuration.

\section{CUDA GPU ACCELERATION}

NAMD can use CUDA to offload work to NVIDIA GPUs for significant acceleration. CUDA binaries, built by passing --with-cuda to the NAMD config script, require an NVIDIA GPU to run and do not support some simulation features. The lack of CPU fall-back is intentional, as it prevents users from inadvertently allocating GPU-accelerated nodes while receiving no benefit from them.

Despite offloading most calculations to the GPU, those remaining on the CPU are increasingly the performance-limiting bottleneck of the simulation, and therefore all available CPU cores should be used. Due to GPU driver inefficiencies, GPUs should never be shared by multiple processes, and therefore the NAMD config script will require an smp (or multicore) Charm++ platform for CUDA builds, and will further not allow mpi-smp due to its historically poor performance. The same Charm++ (smp) libraries can be used for CUDA and non-CUDA builds. NAMD does not use the Charm++ internal CUDA offload features, and therefore Charm++ should not be built with CUDA support enabled.

For simulations running single host it is most efficient to have a single process bind to all GPUs, while for multi-host runs having a separate process per GPU will allow the maximum number of communication threads. NAMD will use Charm++'s physical node awareness to distribute all GPUs among processes on a host, or “+devices $i d[, \ldots]$ " may be used to select specific devices. Note that the device IDs used by the CUDA library (and reported by NAMD at startup for the first two hosts in the run) may not match those reported by nvidia-smi. Job launch utilities on GPU-accelerated clusters may limit the set of GPUs "visible" to each process, e.g., 4 processes running on a host with 4 GPUs may all see only a single device of ID 0 and conclude that the 4 processes are (inefficiently) sharing the same device. If each processes is in fact seeing only its own dedicated device then the +ignore-sharing option can be used, with the desired lack of sharing verified by inspecting the device $\mathrm{PCI}$ addresses reported during NAMD startup. 


\section{MULTI-COPY ALGORITHMS}

NAMD implements a variety of multi-copy (or replica-based) algorithms [2], which employ loosely-coupled simultaneous simulations of the same system to increase sampling and calculate thermodynamic properties of molecules. These are supported efficiently by the Charm++ partitioning feature in LRTS machine layers. When the + partitions $n$ (or + replicas $n$ ) option is specified, the processes are divided equally among the partitions and $n$ independent copies of the application are launched. A process cannot be shared by two partitions, and therefore the number of processes must be a multiple of the number of partitions. The copies may communicate among themselves using low-level Charm++ calls that have been used to implement Tcl scripting commands in NAMD (numReplicas, myReplica, replicaSend, replicaRecv, etc.), enabling user modification and customization of the multi-copy algorithm scripts provided in the NAMD lib directory.

One important limitation of multi-copy support in NAMD is that, for CUDA builds, if processes from different partitions are present on the same host, then each partition will assume that it has exclusive use of all of the GPU devices available (or specified by +devices). To avoid inefficient sharing of GPUs by multiple processes, the +devices-per-replica $n$ option should be used to specify that each partition should use only $n$ GPUs per host, with the assumption that the partitions assigned to each host are numbered consecutively. If it is necessary to run more partitions than GPUs are available then oversubscription is unavoidable, but "+devicesper-replica 1" should still be used to minimized the number of processes sharing each GPU. The nvidia-smi utility can be used to verify the number of processes accessing each GPU.

\section{PARALLEL SCALING}

HPC users should be reminded of the importance of regularly checking the performance of their simulations, particularly at the beginning of an extended campaign. In particular, parallel scaling should be measured for a range of node counts (e.g., powers of two) to select an appropriate trade-off between performance and efficiency.

If scaling efficiency is less than expected, the impact of any special features or options being set should be investigated by comparing performance with and without them. One NAMD feature known to degrade scaling is the Collective Variables (colvars) module, which allows a variety of steering protocols to be applied to the system, but which does most of its additional calculations on the first process of the run. For complex collective variable definitions involving many atoms this work can limit the performance of the simulation even after the load balancer has removed all other work. In addition to reducing the size or complexity of the colvar definitions, performance may also be improved by increasing the number of worker threads per process, i.e., running an smp build of NAMD with a single process per host using all available cores, a configuration that would not be optimal for a simpler benchmark.

When comparing the performance impact of simulation features and options, tests should be run at node counts that provide close to the desired parallel efficiency, with the goal of either increasing performance at the same node count or reducing node count while maintaining performance. Tests should be run multiple times backto-back within the same job to eliminate variation due to different hosts and network topologies, and to a lesser degree variation in network congestion due to other running jobs. If significant performance variation is observed within a run, or between runs in the same job, then it is likely that external factors beyond the user's control, such as operating system noise, are limiting performance and should be investigated further.

\section{OPERATING SYSTEM NOISE}

Tightly coupled parallel applications, such as NAMD, with iteration times on the order of milliseconds are highly sensitive to "noise" that randomly slows or delays even single threads or messages, with the potential performance impact increasing with size of the run. The elimination of application interference by the operating system drove the use of micro-kernels in the first generations of Cray XT and IBM Blue Gene machines, but with full Linux operating systems in use on commodity HPC clusters it is important to monitor or eliminate sources of noise such as service daemons. Cray systems provide a "core specialization" option at application launch, which restricts all system activities to the last core on each node, leaving the remainder clear for the application. On commodity clusters it has long been and continues to be beneficial to leave one core free of application threads, with the expectation that service daemons will migrate to the mostly idle core.

A more recent source of system noise is variable processor clock speeds. NAMD is particularly susceptible to these effects as it employs measurement-based load balancing, for which clock speed variation falsifies the basic assumption that a given task will require the same amount of time in the future, on any core, as it does now. Default "balanced" performance settings may try to adjust the processor clocks to maintain full load, while NAMD is both limited to the performance of the slowest node and suffers from degraded load balancing. Full "performance" mode runs continuously at full speed, but wastes energy when the node is idle. A good compromise is to rapidly increase clock speed when load is present, but to only reduce clock speed slowly when a core is idle.

\section{VERY LARGE SIMULATIONS}

In order to support very large simulations of 1 million to 2 billion atoms, NAMD must be compiled in memory-optimized mode by specifying --with-memopt on the NAMD config line. The resulting binary will require for input a compressed molecular structure generated by a regular (non-memopt) NAMD binary on a large-memory machine. In addition, an smp build of Charm++ should be used to reduce the amount of per-process replicated data on each host, and to reduce redundant communication. Although in use for many years [4], memory-optimized builds should be considered experimental. Users contemplating extremely large simulations should contact the NAMD development team for specialized guidance.

\section{CONCLUSION}

This paper has provided the essential background material needed to support and guide NAMD users in the efficient and productive use of HPC systems. Additional specific and up-to-date information is available on the NAMD website (www.ks.uiuc.edu/Research/namd), in particular in the User's Guide, Release Notes, and Wiki, and also on the Charm++ website (charm.cs.illinois.edu). 


\section{ACKNOWLEDGMENTS}

This research is part of the Blue Waters sustained-petascale computing project, which is supported by the National Science Foundation (awards OCI-0725070 and ACI-1238993) and the state of Illinois. Blue Waters is a joint effort of the University of Illinois at Urbana-Champaign and its National Center for Supercomputing Applications.

This research used resources of the Argonne Leadership Computing Facility, which is a DOE Office of Science User Facility supported under Contract DE-AC02-06CH11357, through the Theta Early Science Program.

This work used the Extreme Science and Engineering Discovery Environment (XSEDE), which is supported by National Science Foundation grant number ACI-1548562, through allocation TGMCB080133.

This paper draws on the author's previous work in NAMD development at the Illinois NIH Center for Macromolecular Modeling and Bioinformatics, supported by the National Institutes of Health grant P41-GM104601, where NAMD development continues in close collaboration with Charm++ development at the Illinois Parallel Programming Laboratory, led by Laxmikant V. Kale.

\section{REFERENCES}

[1] Bilge Acun, Abhishek Gupta, Nikhil Jain, Akhil Langer, Harshitha Menon, Eric Mikida, Xiang Ni, Michael Robson, Yanhua Sun, Ehsan Totoni, Lukasz Wesolowski, and Laxmikant Kale. 2014. Parallel Programming with Migratable Objects: Charm++ in Practice (SC).

[2] Wei Jiang, James Phillips, Lei Huang, Mikolai Fajer, Yilin Meng, James Gumbart, Yun Luo, Klaus Schulten, and Benoit Roux. 2014. Generalized Scalable Multiple Copy Algorithms for Molecular Dynamics Simulations in NAMD. Comput. Phys. Commun. 185 (2014), 908-916. https://doi.org/10.1016/j.cpc.2013.12.014

[3] Laxmikant Kale, Anshu Arya, Nikhil Jain, Akhil Langer, Jonathan Lifflander, Harshitha Menon, Xiang Ni, Yanhua Sun, Ehsan Totoni, Ramprasad Venkataraman, and Lukasz Wesolowski. 2012. Migratable Objects + Active Messages + Adaptive Runtime $=$ Productivity + Performance A Submission to 2012 HPC Class II Challenge. Technical Report 12-47. Parallel Programming Laboratory.

[4] Chao Mei, Yanhua Sun, Gengbin Zheng, Eric J. Bohm, Laxmikant V. Kalé, James C. Phillips, and Chris Harrison. 2011. Enabling and Scaling Biomolecular Simulations of 100 Million Atoms on Petascale Machines with a Multicore-optimized Message-driven Runtime. In Proceedings of the 2011 ACM/IEEE conference on Supercomputing. Seattle, WA, 61:1-61:11.

[5] Juan R. Perilla, Jodi A. Hadden, Boon Chong Goh, Christopher G. Mayne, and Klaus Schulten. 2016. All-atom molecular dynamics of virus capsids as drug targets. F. Phys. Chem. Lett. 7 (2016), 1836-1844. https://doi.org/10.1021/acs. jpclett.6b00517

[6] James C. Phillips, Rosemary Braun, Wei Wang, James Gumbart, Emad Tajkhorshid, Elizabeth Villa, Christophe Chipot, Robert D. Skeel, Laxmikant Kale, and Klaus Schulten. 2005. Scalable Molecular Dynamics with NAMD. f. Comp. Chem. 26 (2005), 1781-1802. https://doi.org/10.1002/jcc.20289

[7] James C. Phillips, John E. Stone, and Klaus Schulten. 2008. Adapting a MessageDriven Parallel Application to GPU-Accelerated Clusters. In SC '08: Proceedings of the 2008 ACM/IEEE Conference on Supercomputing. IEEE Press, Piscataway, NJ, USA. (9 pages).

[8] James C. Phillips, John E. Stone, Kirby L. Vandivort, Timothy G. Armstrong, Justin M. Wozniak, Michael Wilde, and Klaus Schulten. 2014. Petascale Tcl with NAMD, VMD, and Swift/T. In SC'14 workshop on High Performance Technical Computing in Dynamic Languages (SC '14). IEEE Press, 6-17.

[9] James C. Phillips, Yanhua Sun, Nikhil Jain, Eric J. Bohm, and Laximant V. Kalé 2014. Mapping to Irregular Torus Topologies and Other Techniques for Petascale Biomolecular Simulation. In Proceedings of the International Conference on High Performance Computing, Networking, Storage and Analysis (SC '14). IEEE Press, 81-91. https://doi.org/10.1109/SC.2014.12

[10] John E. Stone, Antti-Pekka Hynninen, James C. Phillips, and Klaus Schulten 2016. Early Experiences Porting the NAMD and VMD Molecular Simulation and Analysis Software to GPU-Accelerated OpenPOWER Platforms. International Workshop on OpenPOWER for HPC (IWOPH'16) (2016), 188-206. 